\title{
Posture et imposture de Michel Houellebecq ou le paradoxe du tricheur
}

\section{Sylvie Ducas}

\section{(2) OpenEdition \\ Journals}

Édition électronique

URL : https://journals.openedition.org/fixxion/372

DOI : $10.4000 /$ fixxion.372

ISSN : 2295-9106

Éditeur

Ghent University

Référence électronique

Sylvie Ducas, «Posture et imposture de Michel Houellebecq ou le paradoxe du tricheur », Revue critique de fixxion française contemporaine [En ligne], 22 | 2021, mis en ligne le 15 juin 2021, consulté le 17 février 2022. URL : http://journals.openedition.org/fixxion/372 ; DOI : https://doi.org/10.4000/ fixxion.372

Ce document a été généré automatiquement le 17 février 2022.

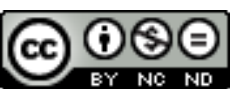

Les contenus de la Revue critique de fixxion française contemporaine sont mis à disposition selon les termes de la licence Creative Commons Attribution - Pas d'Utilisation Commerciale - Pas de Modification 4.0 International. 


\title{
Posture et imposture de Michel Houellebecq ou le paradoxe du tricheur
}

\author{
Sylvie Ducas
}

\begin{abstract}
"Nous sommes des crapules romanesques."
Pierre Michon, Rimbaud le fils, 1991.

"Le monde pue."

Michel Houellebecq, H.P. Lovecraft. Contre le monde, contre la vie, 1990.
\end{abstract}

1 Nul écrivain contemporain ne possède une réputation plus clivée d'auteur à succès que Michel Houellebecq. Figure vénérée comme une icône par les uns ou détestée comme une imposture par les autres, cet auteur cultive à merveille l'ambivalence, et son territoire est le brouillage des cartes. Il incarne une stratégie auctoriale très singulière et très consciente, qui se joue des crises de notre temps et pointe toutes ses dérives menteuses, en faisant entrer en friction et en porosité ses récits et la gestion de son image publique, de sorte que l'on ne sait jamais, dans un tel jeu, à quel saint ou diable se vouer.

2 D'abord parce qu'il pousse à son paroxysme, de toute la force de son charisme incivil, les simulacres médiatico-publicitaires de notre société du spectacle dont il est l'une des plus célèbres incarnations. Ensuite parce qu'il est sans doute le seul écrivain actuel à construire dans un même mouvement sa figure auctoriale et ses récits sur une esthétique et une poétique de l'indécidable dans et hors le livre, propice à toutes les variations interprétatives. Houellebecq le menteur se niche dans ce double-jeu de la persona et de l'œuvre, servi par un art de la provocation qui, en dehors de sa charge publicitaire, sonne vrai et fait mouche à tout coup.

3 C'est ce lien sans cesse cultivé entre une fiction menteuse et un auteur tricheur que l'on se propose d'étudier, en montrant que la mauvaise foi et l'imposture au fondement de toute littérature ${ }^{1}$ construisent avec lui une aire de jeu particulièrement perverse, qui 
déborde le cadre habituel de la fiction pour mieux ouvrir un espace de fiction de "l'homme" et de "l'œuvre" radicalement douteux et louche. Janus bifrons poreux au désenchantement tout schopenhauerien du monde et jouant les Cassandre de pacotille de son devenir funeste, mais aussi bonimenteur de génie, provocateur sans vergogne et figure hilare d'un Moi cultivant l'humour du désastre, Houellebecq berne son public en lui faisant croire, dans sa prose dénuée de toute foi tout comme par sa pose médiatique versatile, au vide métaphysique de notre temps, alors que l'auteur déconstruit, dans des romans pseudo-scientifiques et des pseudo-discours critiques, la littérature elle-même et en manifeste sa haine ${ }^{2}$. Une définition radicalement perverse et névrotique, en somme, de l'écriture et de l'écrivain houellebecquiens : celle d'un virtuose de la triche puisque tout le monde triche, y compris les institutions littéraires et le lecteur.

\section{Le monstre éditorial et (trans)médiatique}

4 Michel Houellebecq est un monstre. Non pas un être dont l'indignité morale serait abjecte, mais une "créature éditoriale" 3 de celles qu'enfantent aujourd'hui les éditeurs quand ils ont flairé et ferré un gros poisson: un auteur de best-sellers. Cet "objet vendeur non identifié" 4 est plus complexe qu'il n'y parait, et son auteur aussi. Quoi de commun, en effet, entre Michel Houellebecq et Katherine Pancol ou Fred Vargas? Même s'ils ne se ressemblent ni par l'écriture ni par les sujets de leurs livres, ils vendent tous en masse des livres pas trop épais pour lecteurs pressés, sont des auteursmarques façonnés comme des produits de luxe et négociés parfois à des millions d'euros par les éditeurs. Mais ces créatures éditoriales tiennent du Frankenstein de Mary Shelley: elles échappent un jour à leurs créateurs qui ont joué les apprentis sorciers avec elles; elles s'autonomisent, renégocient leur image et leur contrat d'édition à mesure que leur succès grandit et leur confère le statut d'icônes sociales.

C'est particulièrement vrai de Michel Houellebecq. De la surmédiatisation - et la monstration spectaculaire qu'elle suppose - à la canonisation, le parcours est sans faute : succès de scandale alimenté par les médias, tractations financières démentielles avec les éditeurs Fayard et Flammarion, un prix Goncourt obtenu en feutrant un peu sa plume après trois échecs successifs ${ }^{5}$, un très légitimant Cahier de L'Herne de son vivant par lequel une certaine université salue en lui un nouveau Balzac, Baudelaire ou Céline. On n'attend plus que son entrée dans la collection de La Pléiade avant le prix Nobel de Littérature... Il a déjà reçu la Croix d'honneur. À l'ère transmédiatique du "je suis partout" pour que "tout le monde en parle", Houellebecq n'hésite pas, en outre, à troquer sa casquette d'écrivain contre d'autres rôles: acteur, réalisateur, musicien, photographe, etc.

6 Sa boulimie transmédiatique et sa consécration littéraire font exploser les hiérarchies de la valeur et dynamitent la ligne de démarcation entre une littérature académique dite légitime et une littérature de masse friande de produits culturels qui répondent à leurs attentes. Houellebecq devient surtout l'écrivain-symptôme d'un effondrement du monde littéraire, de ses paillettes et déchets, mais aussi de la lecture et du jugement critique, dans nos sociétés de loisir jusqu'aux instances les plus académiques du goût. Et si l'industrie du succès littéraire ou culturel concurrence aujourd'hui le marché du sexe et de l'argent, cette triste trinité de nos sociétés malades du capitalisme, Houellebecq en fait ses choux gras, dans la construction de son image publique comme dans ses livres. 
7 Il est cet auteur "monstre" au succès monstre qui a tiré les leçons de "la société du spectacle" théorisée par Guy Debord : politique et marché libéral ont la publicité pour outil totalitaire de propagande. Alors Houellebecq n'hésite pas à faire de son corps une œuvre d'art aux airs de produit promotionnel périmé. Son incarnation auctoriale n'est qu'un jeu d'apparences se donnant pour vraies, qu'il manœuvre à loisir sans qu'on puisse jamais saisir Michel derrière Houellebecq: parka fatiguée, cheveux plats, chemise terne sur pantalon terne cachant un corps flottant, voix faible et regard fuyant, cigarette qui se consume, tous désespérément ordinaires et condamnés à une obsolescence programmée largement décrite dans La carte et le territoire. Pourtant le "phénomène Houellebecq" perdure car l'image ou l'apparence qui vaut pour essence et vérité aujourd'hui, cet auteur l'incarne comme nul autre: Protée médiatique, il joue à souhait d'un corps inconsistant, virant de l'écrivain propret à l'atrabilaire déprimé au gré des humeurs et de l'actualité ; corps-avatar qu'il construit parfois avec l'aide de sa maquilleuse et de sa coiffeuse, sans apprécier, bien sûr, qu'on ne révèle de telles coulisses. Toute imposture auctoriale avance masquée, on le sait bien. Celle de Houellebecq vise à "maquill[er] une impuissance en triomphe" 6 , comme dirait Pierre Michon. Son corps minable érigé en icône d'une époque minable pourrait faire croire à un auteur génial, de même que moins par moins égale plus en mathématiques. Mais l'ambition houellebecquienne vise surtout à exhiber une "momie de l'auteur" qui, après "la mort de l'auteur", n'est plus qu'une surmarionnette à la Genet d'une mort en marche et la mise en scène du corps social de l'écrivain, un corps à l'agonie comme dans du mauvais body art, ou pire, dans du pornhub. À ce titre, le pastiche hilarant à la Bret Easton Ellis du meurtre de l'écrivain Houellebecq dans La carte et le territoire ${ }^{7}$ est savoureux dans sa façon de figurer "la mort de l'auteur" barthésienne en ayant recours aux ressorts les plus gore du thriller horrifique.

8 Toutefois, ce comique “à grincer des dents" ${ }^{8}$ 'empêche pas la mauvaise foi: Houellebecq nous tend dans ses livres et hors d'eux le miroir grimaçant d'un monde néo-libéral et médiatico-publicitaire dont il est l'une des figures éditoriales les plus lucratives et l'un des acteurs qui en jouit le plus. Il faut donc imaginer Frankenstein cupide et pas si malheureux que cela. Houellebecq ou l'art de faire oublier qu'il est partie prenante du monde qu'il (d)écrit, voilà où se niche la mauvaise foi : dans cette intention cachée, celle d'échapper à ce qu'on critique par une écriture qui absout son narrateur aux yeux des lecteurs et fait oublier le Frankenstein malhonnête qui tient le stylo ou tapote son clavier.

9 Extension du domaine de l'auteur-marque : la trouvaille de Houellebecq est de faire de ce corps médiatisé un corps-métaphore de ses textes, une émanation ou une continuation des apparences tristes qu'il nous donne à lire. En créant des porosités entre son personnage d'auteur et son narrateur, Houellebecq joue, comme dans son image publique, de l'ambivalence et du pouvoir flottant des apparences médiatiques. Ce narrateur apathique et médiocre qui lui ressemble et dans lequel certains lecteurs se reconnaissent aussi sans doute, c'est monsieur-tout-le-monde, autrement dit personne : “Je viens d'avoir trente ans. Après un démarrage chaotique, j'ai assez bien réussi dans mes études: aujourd'hui, je suis cadre moyen. Analyste-programmateur dans une société de services en informatique, mon salaire net atteint 2,5 fois le SMIC; c'est déjà un joli pouvoir d'achat" ; ou Michel, "fonctionnaire blasé de 40 ans, [...] part[ant] en Thaillande pour y goûter aux plaisirs exotiques" ${ }^{10}$. Médiocrité ordinaire de ce Droopie neurasthénique ou de ce narrateur-auteur-zombie ${ }^{11}$ qui, dans et hors le 
livre, "port[e] cette armature rigide, l'apparence", aurait dit Victor Hugo. "Il était monstre en dessous; il vivait dans une peau d'homme de bien avec un cœur de bandit"12. Avec lui, Frankenstein est donc un escroc.

\section{Plagiaire et pilleur de cadavres : un Lovecraft des existences ordinaires}

10 Michel Houellebecq est aussi un plagiaire, même si l'on sait bien que tout écrivain l'est $^{13}$. En bon rat de bibliothèque, il fait paradoxalement du pastiche et de l'emprunt son cheval de Troie dans la forteresse de la consécration littéraire dont il entend bien abattre toutes les murailles, la plus épineuse étant celle de la singularité de plume. Or, Houellebecq ne pratique pas que le clin d'œil entendu : il pille. Mais n'est pas pillard de génie qui veut. Si ses pages témoignent d'un recours convenu à l'intertextualité, par laquelle tout auteur se rachète d'être un lecteur jaloux de celui qui a été génial avant lui, le génie houellebecquien tient au fait que son pillage réel est invisible, caché derrière un jeu de pistes contrôlé vers des références trop facilement visibles et relayées massivement par des médias qu'il déteste. Houellebecq sait bien que trop d'emprunts tue l'emprunt, de même que l'auteur-Wikipédia n'a pas un grand avenir devant lui. Houellebecq, lui, veut être le meilleur dans l'industrie des meilleures ventes, le seul à réussir à transcender sa valeur marchande en valeur littéraire ou du moins à faire en sorte que l'on confonde les deux. Pour cela, la bibliothèque et sa Babel de livres sont un outil précieux. Il connaît surtout très bien la tactique de diversion qui vise à induire en erreur les lecteurs en leur cachant ses intentions profondes pour mieux les en détourner. Houellebecq cultive le syndrome des objets brillants, cher au marketing, cette prestidigitation pour distraire son public et dissimuler sa tromperie. Il livre ainsi toutes ses influences de lecteur autodidacte et boulimique pour mieux cacher aux lecteurs sa vraie source profonde, concentrés qu'ils sont sur la baguette magique, pour ne pas voir le lapin glissé dans le chapeau. Certes, Huysmans, Balzac, Auguste Comte, Schopenhauer, les manuels de physique quantique, Dostoïevski, Baudelaire, les cartes Michelin se bousculent sur l'autel des maîtres houellebecquiens. Mais il est une référence profonde, une matrice première de l'écriture à laquelle il a consacré un petit essai passionnant ${ }^{14}$ pour ne plus beaucoup en parler ensuite, mais qui court pourtant dans toute son œuvre comme un accélérateur de particules : c'est celle de Lovecraft.

Cet essai de jeunesse que lui consacre Houellebecq avant d'entrer en écriture peut être lu comme un véritable art poétique de sa propre œuvre, le pillage de l'un justifiant les partis pris littéraires de l'autre. Houellebecq y décortique la machine à faire peur lovecraftienne pour délimiter son propre territoire. Non pas qu'il s'agisse seulement d'imiter ses intrigues horrifiques, mais de détourner cette matrice première de SF vers des thèmes délaissés par Lovecraft : le sexe, l'argent, le monde actuel. Houellebecq va ainsi créer une dystopie à la fois terrifiante et navrante de nos existences ordinaires, dans laquelle les dérives capitalistiques accoucheraient d'un monstre rappelant le vieux mythe de Cthulhu, fondé sur les pires craintes de l'humanité. Le monde de Lovecraft, on le sait, est un reflet effrayant du monde réel; celui de Houellebecq en est un autre, qui fait, cette fois, de l'infra-ordinaire le cadre d'un scénario-catastrophe, sans cosmogonie comme chez Lovecraft, mais tout aussi terrifiant car sans échappatoire, sinon des îlots utopiques sombrant lamentablement dans "l'impossibilité d'une île": le sexe heureux, la science, l'art. Et même boîte à outils littéraire que son modèle pour 
parvenir à (d)écrire ce désastre horrifique : matérialisme mécaniste, platitude voulue des personnages, dissonance cognitive qui les frappe, caution du discours scientifique et des reflets documentaires qu'il crée dans le récit, sentiment d'"épouvante objective" ${ }^{15}$ à placer l'inimaginable au cœur de notre existence ordinaire, à rendre l'inconcevable possible, à "dépasser les bornes"16 et créer ainsi un monde plausible pour dire ce qui n'est pas. Halluciner le réel, en somme. Et créer un dispositif d'écriture pervers visant, pour y parvenir, à créer une "impression accrue de vraisemblance en jouant une référentialité pseudo-documentaire" ${ }^{17}$. Le néo-naturalisme ou le positivisme des récits houellebecquiens réussissent ainsi à faire oublier qu'on ne lit qu'un système sémiotique, non pas une réalité saisie, comme chez Stendhal, par un "miroir au bord du chemin", et c'est un narrateur perspicace qui embarque ainsi le lecteur et lui impose sa vision thanatographique. Pour ne donner qu'un exemple, la ville et sa banlieue chez Houellebecq sont atroces : l'espace péri-urbain, notamment, avec ses ronds-points et ses délaissés miteux, est fait de lieux sans qualité, avec leurs hypermarchés et leurs zones commerciales qui surgissent dans leur vide architectural, symbole d'un monde définitivement médiocre et à l'agonie. Un Paris qui tue le tissu social et aurait perdu sa capitale comme on aurait perdu la tête. Le choc hyperréaliste tient à ce mélange savant d'observation désenchantée et de monstration clinique ; pourtant, "l'écriture est plate, souvent ironique, pointilleuse et encyclopédique, elle colle à la banale médiocrité de la France urbaine au point de n'être plus qu'un tissu de lieux communs partagé par l'imaginaire collectif" ${ }^{\prime 1}$. Assimiler le discours de la doxa à de la littérature - ce pas de côté qui l'interroge et la réfute -, c'est mentir au lecteur, lui faire croire que le petit noyau de vérité des stéréotypes exploités par l'auteur est la vérité majuscule, alors que ce ne sont que des clichés sans objectivité scientifique, à l'ère du brouhaha médiatique des fake news.

12 Pour Houellebecq, il s'agit donc de repérer et d'exploiter les peurs du lecteur, de refléter dans ses livres ce que le lecteur a dans la tête. Rien d'une prédiction dans sa démarche, mais une invention de fictions réalistes et plausibles à partir de ces peurs. Et quel plus gros mensonge que d'abuser son lecteur en lui faisant prendre des croyances pour des documents, des craintes pour des preuves? Que le dispositif narratif soit mou en fait paradoxalement la force: de fait, le ton morne et badin avec lequel dans Soumission il imagine dans un futur proche ${ }^{19}{ }^{1}$ 'effondrement du système politique à la française et la montée d'un islam modéré, est d'une efficacité bien plus insidieuse que la dystopie violente d'un totalitarisme islamiste planétaire que Boualem Sansal prédit dans $2084^{20}$. Car dans cette "auberge espagnole ouverte à tous les vents de l'angoisse française contemporaine" ${ }^{21}$, le récit houellebequien fait sortir ce mauvais rêve de l'islamisation comme un diable au milieu de la blanquette de veau Casino, de BFM-TV, des femmes si vite périmées et d'un essai ennuyeux sur Huysmans, qui moisissent dans le cerveau de son "pauvre petit blanc"22 de personnage principal qui lui ressemble tant. La stratégie militaire de la surprise, celle qui frappe là où l'ennemi ne l'attend pas, comme on s'amuse à se faire peur pour Halloween, Houellebecq en use et abuse à l'égard de son seul véritable adversaire : le lecteur.

\section{Manipulation du lecteur, "houellebecquisé"}

13 Michel Houellebecq est un manipulateur qui "houellebecquise" la réception de ses livres. Comme pour tout écrivain, la seule particule élémentaire qui compte pour lui, 
c'est la mécanique quantique de l'écriture à la lecture. Comme tout écrivain, Houellebecq n'en contrôle pas le champ de forces, d'où des liens nuls avec ses réfractaires, des liens faibles avec les sceptiques, des liens forts avec ses fans. Mais dans chacun de ses livres, il y a à boire et à manger (ou vomir) pour tous. Mais que recherchent ces victimes consentantes qui acceptent d'être "houellebecquisées" et en redemandent. Sur quel contrat d'imposture dévorent-elles du Houellebecq ? À peu près le même qui faisait le talent oratoire d'un Le Pen: un jeu sur nos peurs, un langage simple, des clichés.

14 L'écriture doxologique de Houellebecq construit, en effet, au fil des livres - pour atteindre des sommets dans Sérotonine -, un vaste défouloir de tout ce qui pourrit dans l'inconscient collectif, que la morale réprime et qui lui répugne : sexisme, homophobie, racisme, zoophilie. "L'hystérisation absolue des signes houellebecquiens" ${ }^{23}$ dans Sérotonine, par exemple, pourrait faire sourire le bourgeois lettré s'il n'y éprouvait le malaise que suscite toute banalisation du mal, toute jubilation de l'auteur à faire entrer en littérature la bêtise réactionnaire la plus primaire, tout en prenant ses distances par rapport à elle puisqu'il n'est pas prouvable qu'il soit le narrateur et que tout n'est que fiction blagueuse, provocation pour rire. En se défaussant ainsi, Houellebecq fait mal à la posture de l'écrivain, à son ethos clinquant de penseur engagé et à sa responsabilité en débat aujourd'hui ${ }^{24}$ sur fond de pédophilie - comme un symptôme de plus d'une époque qui préfère toucher le corps social de l'écrivain et ses stigmates purulents que lire ses livres. Il renvoie le texte à ses pouvoirs performatifs comme le lecteur à sa lecture moralisante ou sans nuance. Le seul coupable, Gros Jean comme devant, c'est donc le lecteur, trahi par l'auteur dans le contrat "win-win" de toute lecture, celui d'une confiance et d'une philia réciproques.

Car le vrai problème que pose Houellebecq n'est pas la poétique ou l'esthétique de ses textes, mais celui de leur réception. Avec pour corollaire obligé au doxique, l'émotion : qu'elle soit négative ou positive, elle est centrale chez Houellebecq pour faire accéder les lecteurs à "de nouvelles représentations du monde" 25 par la technique éprouvée $d u$ "Mettez le doigt sur la plaie et appuyez bien fort !"

On se souvient de Roland Barthes, dans ses derniers cours au Collège de France : "il faut se rendre à l'évidence : toutes choses sont lues au premier degré; la simplicité veut, voudra donc qu'on écrive le plus possible au premier degré" ${ }^{27}$. Houellebecq écrit au premier degré et le public se fait piéger par ce même degré premier de la lecture. Les effets d'actualité (plus que de réel) dans ses textes sont, notamment, d'une pernicieuse efficacité. Bien sûr, Houellebecq ne les contrôle pas, mais il n'hésite pas à se construire une posture et un destin de victime, malgré lui, de son don présumé de prophétie. Au moment de l'étrange collision entre la sortie de Soumission et l'attentat de Charlie Hebdo, il déclare ainsi ${ }^{28}$ :

Ça donnerait à croire qu'effectivement il y a une divinité qui crée des conjonctions d'événements et que je suis entraîné dans ce que l'on appelle un destin. [...] C'est un peu effrayant. Ça ne me donne pas une idée de responsabilité, ça me donne une idée que je suis manipulé par une divinité maléfique. C'est très désagréable.

17 Et comme ces télescopages se répètent, ils renforcent, de manière virale, ce statut de romancier voyant ou de détecteur de vérités sociales qu'on attribue à l'auteur de Plateforme, de Soumission et de Sérotonine. D'où la dérive ubuesque et inquiétante d'une lecture ordinaire vers la croyance en la prophétie messianique d'un écrivain en habits de gourou trop grands pour lui. 
Pourtant, l'écriture houellebecquienne n'affirme rien; elle est, paradoxalement, indécidable. Sans que l'on soit certain que cette indécidabilité soit un effet de style (la fameuse "mise en abyme de l'indécidabilité" 29 du monde) ou bien une dérobade d'auteur laissant au lecteur la patate chaude du sens à y voir et rompant le pacte de lecture d'une "logique du sens" 30 que tout lecteur voudrait partager avec l'auteur.

Indécidabilité et ambiguïté, donc, d'une écriture aussi plate que l'électrocardiogramme d'un cadavre, mais qui fait parfois frémir l'antithèse osée ("le sommet du bonheur humain réside dans la soumission la plus absolue" ${ }^{31}$; "Tout peut arriver dans la vie, et surtout rien" ${ }^{\prime 2}$...) et s'autorise quelques hoquets de pathos poétique, voire de lyrisme souvent maritime ("la mer scintillait, réfractait une lumière mobile sur les derniers îlots rocheux" ${ }^{33}$; "puis tout se calme, il n'y a plus que des herbes agitées par le vent. Le triomphe de la végétation est total" ${ }^{34}$...). Frankenstein serait-il fleur bleue ou mauvais poète ou se paie-t-il tout simplement notre tête?

Mais c'est précisément dans cette "impassibilité d'un style" ${ }^{35}$ que Houellebecq est redoutable, d'autant qu'elle s'allie à un humour tantôt désenchanté tantôt grivois ou à une ironie grinçante qui est une vraie marque de fabrique houellebecquienne et une méthode de Sioux effaçant les traces de ses pas derrière lui pour tromper l'ennemi. Puissant désamorceur d'illusion référentielle, l'humour est, certes, aussi désamorceur d'angoisse, celle du lecteur englué malgré lui, le reste du temps, dans la position inconfortable de "gardien de but au moment du pénalty"36 ("Tout cela me donnait un peu envie de mourir, mais je me contins" ${ }^{37}$; “N'ayez pas peur du bonheur, il n'existe pas" $\left.{ }^{\prime 3} . ..\right)$; il est enfin ce facteur d'incertitude et de bipolarité dépressive qui brouille les cartes et ouvre un champ étonnamment contradictoire d'interprétations idéologiques des textes et de la posture de leur auteur : tour à tour jugé réactionnaire, anarchiste, dandy décadent, catholique, nihiliste, humaniste, escroc commercial, génie, opportuniste...

21 Cet écrivain sans engagement clair, ni sur les plateaux télé ni dans ses livres, multipliant les reculades au gré des fluctuations hasardeuses de particules élémentaires, se défausse sans cesse, écrit comme il pose ${ }^{39}$, en clone ou clown protéiforme mimant les comportements contemporains de ceux qui le lisent. Devant le Frankenstein houellebecquien, le lecteur n'en a que davantage le doigt sur la couture et en redemande. La force de conviction de ces fictions molles est pourtant une violence faite à la littérature.

\section{Haine de la littérature : le paradoxe du tricheur}

Michel Houellebecq n'aime pas la littérature. Non pas qu'il crache sur elle, au contraire, on l'a dit, il la pille. Mais surtout il la détruit. Pas par simple réaction de vengeance face à la "rage de ne pas lire" 40 des lecteurs dénoncée par Dominique Noguez, ni de haine atrabilaire de ne pouvoir "rester vivant" qu'en se réfugiant dans les mots et la fiction.

Houellebecq n'aime pas la littérature parce qu'il n'aime pas les lecteurs qui la lisent. Alors il détruit toute illusion sur les présumés bénéfices de la fiction littéraire auxquels on nous a longtemps fait croire : ses vertus moralement édifiantes, son utilité dans nos vies, son pouvoir d'empathie qui rend meilleur le lecteur. Emma Bovary et Don Quichotte nous ont, certes, appris les dangers qu'il y a à se perdre dans la fiction et à prendre des vessies pour des lanternes. Mais quand on lit, c'est toujours avec le désir de 
se lier à un autre capable de nous prendre la main et de mettre des mots, ironiques ou non, sur notre Dasein angoissé. Et pour se lier à l'auteur, il faut lui faire confiance. Or, Houellebecq n'est digne d'aucune confiance: "crapule romanesque" comme tout écrivain, et "professeur de désespoir" ${ }^{41}$ hors pair, la pire forme de ce désespoir étant de se moquer des pouvoirs de la littérature à le surmonter, ce désespoir, et de lui faire la peau, à la littérature.

Certes l'écriture n'a pas pour vocation de divertir, comme cherchent à le faire croire notre culture consumériste et son "opium du peuple" numérique, mais le lecteur aime à y trouver évasion ou consolation. La littérature houellebecquienne ne répare pas ni ne console : elle tire à vue et démolit. Sans aucun salut possible, ni pour le lecteur ni pour la littérature. Le premier, si Houellebecq le séduit, se prend les pieds dans un filet spéculaire et nauséeux qui lui renvoie l'image de ses propres névroses et pulsions de mort. Et s'il se défie de lui, en lecteur averti et rompu, tel un sportif de haut niveau, à la lecture critique et distanciée, il achoppe sur l'angoisse d'un dispositif textuel dénonçant la fiction comme imposture et machine infernale à créer du vent, aussi vaine que les représentations du monde dont la doxa nous a gavés depuis le berceau et désormais balayées par le vent. La littérature s'effondre donc dans la vanité de sa propre imposture face à un monde lui-même aussi menteur et vain que celui qui l'écrit et celui qui la lit. Le texte houellebecquien est cette métastase du néant qui courtcircuite les clivages du savant et du populaire, du légitime et de l'illégitime, du sérieux et du comique, par lesquels on oppose habituellement la lecture courante et la savante. Car il opère - on l'a dit - dans cette zone troublante et hypnotique de la première lecture, celle au premier degré, et joue de ce "sentiment océanique" de dilution des identités propre à la lecture quand la séduction littéraire opère ${ }^{42}$. Dans un tel dispositif, le style importe peu. Seule compte la force de frappe de la représentation littéraire. Elle n'est plus démonstration, mais simple monstration chez Houellebecq. Il suffit que l'auteur énumère en les pointant les signaux forts ou faibles de notre trivialité désastreuse pour les rendre crédibles et se faire passer pour un maître de la clairvoyance. Il ne s'agit plus de représenter ce qui est ou ce qui fut mais de présenter ce qui est et ce qui pourrait être ou advenir. Avec ce petit décalage ou pas de côté pervers du type "Et si l'on voyait là... Et que se passerait-il si... ?" au service d'une réalité en puissance qui fait oublier qu'elle n'est qu'une fabrication du "réel", une simple aire de jeu.

C'est cet univers spéculaire du pseudo, du faux donné pour vrai grâce au tour de magie $\mathrm{du}$ lieu commun, et au tour de passe-passe entre surcontextualisation (les effets d'actualité) et de décontextualisation (les envolées théoriques), qui fait mouche. La ruse consiste à faire croire que l'auteur écrit sans prisme, alors qu'il les démultiplie jusqu'à perdre le lecteur dans cette galerie des glaces. C'est là que se joue ce que j'appelle "l'art de la triche" de Houellebecq: se faire passer pour un démiurge diabolique, quand il n'est - comme tout écrivain - qu'un escroc de la fiction presque vraie dont le but pour Houellebecq est de coincer un présent social anxiogène entre un futur vague, aussi fragile que du carton-pâte, et un passé définitivement trépassé. Autrement dit, inventer cette fable prétendue vraie pour mieux instiller ce "sentiment d'imposture" 43 face au monde qu'on éprouve à le lire : sentiment de ne pas y être à sa place, pas à la hauteur, de jouer un rôle usurpé et illégitime que doit sans doute éprouver l'auteur lui-même et qu'il inocule au lecteur pour mieux masquer l'escroquerie dont il se sent coupable. En faisant ainsi porter le chapeau du magicien et du lapin caché au lecteur, il abandonne ce dernier aux seuls images et reflets morbides qu'il lui tend. Le Frankenstein 
houellebecquien est, finalement, ce "salaud", au sens sartrien de mot, manquant de sérotonine, dont le but est de nous faire croire que sa dépression et la nôtre sont la conséquence de notre société malade à crever ${ }^{44}$. Et il faut peu aimer son lecteur pour lui communiquer pareille croyance.

Et tel est bien le paradoxe du tricheur Houellebecq : ne pas être pris pour un individu malhonnête détournant les règles à son seul profit car c'est le monde entier qui triche partout et tout le temps. Être pris pour un tricheur trompant un monde qui triche frôle même le comble de l'honnêteté. Il y a de la force créatrice à relever pareil défi. Car toute l'énergie de Houellebecq se niche bien là et vise ce seul but : être pris pour un créateur, un écrivain, capable de ce grand détournement du sens commun et des fictions menteuses sur lesquelles il se fonde. Faire même - pourquoi pas? - de son œuvre le lieu d'une forme de progrès humaniste et moral face à un monde social (et littéraire) en pleine désagrégation. Il y a fort à parier, d'ailleurs, que les prochains livres de Houellebecq creuseront le filon de la religion et consolideront sa figure messianique pour mieux sublimer le portrait de l'artiste en affabulateur et en névrosé qui lui colle à la peau.

Alors, bien sûr, reste la possibilité de ne pas lire Houellebecq. Ou bien de prendre directement une pilule de Prozac. Toujours mieux que de partager des fictions menteuses entre auteur tricheur et lecteur complice. Quoique...

\section{NOTES DE FIN}

1. Maxime Decout, En toute mauvaise foi, Paris, Minuit, 2015, <Paradoxe> et Pouvoirs de l'imposture, Paris, Minuit, 2018, <Paradoxe>.

2. William Marx, La haine de la littérature, Paris, Minuit, 2015, <Paradoxe>.

3. Un de mes étudiants de l'UPEC, Nicolas Bailly, termine actuellement sous ma direction un mémoire de Master sur ces "créatures éditoriales" construites de toutes pièces par les éditeurs à l'ère de la best-sellerisation littéraire. C'est à lui que j'emprunte la formule.

4. Sylvie Ducas, "Introduction", in Olivier Bessard-Banquy, Sylvie Ducas et Alexandre Gefen (dirs), Best-seller : l'industrie du succès, Paris, Armand Colin, 2021, p. 11.

5. Régulièrement cité depuis dix ans, Michel Houellebecq avait "raté" le Goncourt en 1998 avec Les particules élémentaires; en 2001, Plateforme, son troisième roman, n'avait pas dépassé la troisième sélection et en 2005 , La possibilité d'une île échoue à une voix près. Pour son cinquième roman, La carte et le territoire, la critique est quasi-unanime et le Goncourt lui est attribué par sept voix contre deux pour le roman de Virginie Despentes, Apocalypse bébé.

6. Pierre Michon, Le roi vient quand il veut. Propos sur la littérature, Paris, Albin Michel, 2007, p. 24.

7. Michel Houellebecq, La carte et le territoire, Paris, Flammarion, 2010, p. 287-291.

8. Jean-Paul Sartre, Saint Genet, comédien et martyr, Paris, Gallimard, 1952, p. 676-677.

9. Michel Houellebecq, Extension du domaine de la lutte, Paris, J'ai Lu, 1997 [1994], p. 15.

10. Quatrième de couverture de Plateforme, Paris, Flammarion, 2001.

11. Paul Vacca, Michel Houellebecq, phénomène littéraire, Paris, Robert Laffont, 2019, <Nouvelles mythologies >, p. 65 .

12. Victor Hugo, Les travailleurs de la mer, 1866. 
13. Antoine Compagnon, La seconde main ou le travail de la citation, Paris, Seuil, 1979; Michel Schneider, Voleurs de mots. Essai sur le plagiat et la psychanalyse, Paris, Gallimard, 2011 [1985], $<$ Tel> ; Maxime Decout, Qui a peur de l'imitation?, Paris, Minuit, 2017, <Paradoxe>.

14. Michel Houellebecq, H.P. Lovecraft : contre le monde, contre la vie, Paris, J'ai lu, Éditions du Rocher, 1999 [1991].

15. Ibid., p. 95.

16. Ibid., p. 110.

17. Marc Attalah, "Les utopies de Michel Houellebecq : hybridation générique et poétique de l'ailleurs", Res Futurae, 8, 2016, mis en ligne le 28 décembre 2016, consulté le 13 mars 2020, disponible sur: <https://journals.openedition.org/resf/877>.

18. Sylvie Ducas, "Fouiller les poubelles et lire les déchets du Grand Paris", in Maria de Jesus Cabral, Maria Herminia Laurel et Franck Schuerewegen (dirs), Lire les villes, Paris, éditions Le Manuscrit, 2020, <Exotopies>, p. 209-210.

19. L'intrigue du livre se déroule en 2022.

20. Les deux ouvrages sont sortis la même année, en 2015.

21. Philippe Lançon, "Houellebecq et le Coran ascendant", Libération, 2 janvier 2015, https:// www.liberation.fr/livres/2015/01/02/houellebecq-et-le-coran-ascendant_1173203/, consulté le 13 mars 2020.

22. Sylvie Laurent, Pauvre petit blanc, Paris, éditions de la Maison des sciences de l'homme, 2020.

23. Johan Faerber, "Houellebecq: Homais romancier (Sérotonine)", Diacritik, 8 janvier 2019, consulté le 28 janvier 2021, disponible sur: <https://diacritik.com/2019/01/08/houellebecqhomais-romancier-serotonine/>.

24. Gisèle Sapiro, La responsabilité de l'écrivain, Paris, Seuil, 2011 ; Peut-on dissocier l'œeuvre de l'auteur?, Paris, Seuil, 2020.

25. Michel Houellebecq, Rester vivant et autres textes, Paris, Librio, 1998 [1991], p. 25.

26. Ibid., p. 26.

27. Roland Barthes, La préparation du roman I et II : cours et séminaires au Collège de France (1978-1979 et 1979-1980), Paris, Éditions du Seuil, 2003, p. 380.

28. Dans l'émission de Ruquier, On n'est pas couché, 29 août 2015.

29. Paul Vacca, op. cit., p. 113.

30. Gilles Deleuze, Logique du sens, Paris, Minuit, 1969, <Critique>.

31. Michel Houellebecq, Soumission, Paris, Flammarion, 2015, p. 260.

32. Michel Houellebecq, Plateforme, op. cit., p. 200.

33. Michel Houellebecq, Les particules élémentaires, Paris, J'ai lu, 2000 [1998], p. 303.

34. Michel Houellebecq, La carte et le territoire, op. cit., p. 428.

35. Paul Vacca, op. cit., p. 67.

36. Allusion au roman de Peter Handke, L'angoisse du gardien de but au moment du penalty (1972)

37. Michel Houellebecq, La possibilité d'une île, Paris, J'ai lu, 2012 [2005], p. 94.

38. Michel Houellebecq, Rester vivant, op. cit., p. 21.

39. Voir Paul Vacca, op. cit., p. 112-113.

40. Dominique Noguez, Houellebecq, en fait, Paris, Fayard, 2003, p. 73.

41. Nancy Huston, Professeurs de désespoir, Arles, Actes Sud, 2004.

42. Jean-François Vernay, La séduction de la fiction, Paris, Hermann, 2019.

43. Pauline Rose Clance, Le complexe d'imposture, trad. de l'américain par Martine Laroche, Paris, Flammarion, 1986; Philippe Di Folco, Petit traité de l'imposture, Paris, Larousse, 2011, $<$ Philosopher> ; Belinda Cannone, Le sentiment d'imposture, Paris, Calmann-Lévy, 2005, <Petite bibliothèque des idées>.

44. Alain Ehrenberg, La fatigue d'être soi. Dépression et société, Paris, Odile Jacob, 1998. 


\section{RÉSUMÉS}

À travers toutes les controverses autour desquelles s'est construite, de manière très clivée, sa figure d'écrivain à succès, figure vénérée comme une icône ou détestée comme une imposture, Michel Houellebecq incarne une stratégie auctoriale très singulière, qui se joue des crises de notre temps et grossit le trait de toutes ses dérives menteuses, en faisant notamment entrer en friction de façon tout à fait inédite ses récits et la gestion de son image publique. Il pousse à son paroxysme, en jouant avec toute la force de son charisme incivil, les simulacres médiaticopublicitaires de notre société du spectacle et en en tirant profit. Et il est sans doute le seul écrivain actuel à construire dans un même mouvement sa figure auctoriale et ses récits sur une poétique de l'indécidable dans et hors le livre, propice à toutes les incertitudes interprétatives.

C'est ce lien sans cesse cultivé entre une fiction menteuse et une figure auctoriale tricheuse que l'on se propose d'étudier. Janus bifrons poreux au désenchantement du monde ou jouant les Cassandre de pacotille de son devenir funeste, Houellebecq berne le lecteur en lui faisant croire qu'il lit, dans sa prose vidée de toute foi tout comme dans sa provocation idéologique, le vide métaphysique de notre temps, alors que l'auteur déconstruit, dans des romans pseudosociologiques, la littérature elle-même et en manifeste sa haine. Une définition possible, en somme, de la mauvaise foi d'un écrivain tricheur puisque tout le monde triche, y compris le lecteur.

\section{INDEX}

Mots-clés : posture, triche, ambivalence, auctorialité, fiction, réception

\section{AUTEUR}

\section{SYLVIE DUCAS}

Université Paris-Est Créteil 